\title{
Gemcitabine in combination with paclitaxel for advanced soft-tissue sarcomas
}

\author{
AMIR SONNENBLICK $^{1}$, FERAS ELEYAN ${ }^{1}$, TAMAR PERETZ $^{1}$, INNA OSPOVAT ${ }^{2}$, \\ OFER MERIMSKY ${ }^{2}$, TAMAR SELLA ${ }^{3}$, NILI PEYLAN-RAMU ${ }^{1}$ and DANIELA KATZ ${ }^{1}$
}

\author{
${ }^{1}$ Sharett Institute of Oncology, Hadassah-Hebrew University Medical Center, Jerusalem 91120; ${ }^{2}$ Department of Oncology, \\ The Tel-Aviv Sourasky Medical Center, Sackler Faculty of Medicine, Tel-Aviv University, Ramat Aviv 69978; \\ ${ }^{3}$ Department of Radiology, Hadassah-Hebrew University Medical Center, Jerusalem 91120, Israel
}

Received December 31, 2014; Accepted February 20, 2015

DOI: $10.3892 / \mathrm{mco} .2015 .545$

\begin{abstract}
A limited number of chemotherapeutic agents have been found to be active against advanced soft-tissue sarcomas (STSs), particularly sarcomas that have progressed following doxorubicin treatment. The aim of this retrospective study was to determine the response to treatment with gemcitabine plus paclitaxel in patients with STSs. Data were collected on all patients with advanced non-resectable STS who were treated with a fixed dose $700 \mathrm{mg} / \mathrm{m}^{2}$ gemcitabine in combination with $70 \mathrm{mg} / \mathrm{m}^{2}$ paclitaxel on days 1 and 8 every 3 weeks. A total of 30 patients were included, with a median age of 56.4 years (range, 40-70 years). The gemcitabine/paclitaxel combination was well tolerated, with an overall response in $27 \%$ and a clinical benefit in $57 \%$ of the patients. The median progression-free survival was 6.1 months and the overall survival was 14.3 months. In conclusion, gemcitabine plus paclitaxel was found to be tolerable and effective in patients with advanced STSs.
\end{abstract}

\section{Introduction}

Soft-tissue sarcomas (STSs) are a heterogeneous family of malignancies originating from mesenchymal tissues. Patients who present with advanced-stage STS or develop disease recurrence following initial resection carry a poor prognosis, since the majority of chemotherapeutic agents have not achieved any survival benefit in this disease. However, $30 \%$ of patients treated with doxorubicin achieve an objective response and the response rates may increase to $35-40 \%$ when doxorubicin is combined with ifosfamide $(1,2)$. The combination of gemcitabine and docetaxel was also proven to be effective in advanced STS, particularly in patients with uterine leiomyosarcoma (LMS) (3). While single-agent gemcitabine exhibited

Correspondence to: Dr Daniela Katz, Sharett Institute of Oncology, Hadassah-Hebrew University Medical Center, Kiryat Hadassah, POB 12000, Jerusalem 91120, Israel

E-mail: danielakatz@hadassah.org.il

Key words: gemcitabine, paclitaxel, soft-tissue sarcomas, stage 4 only modest activity, the overall response rates in phase II trials combining gemcitabine with docetaxel were in the range of $16-53 \%$ in this patient group (3-6). A higher efficacy with superior progression-free survival (PFS) and overall survival (OS) was also demonstrated with this combination in several comparative trials $(3,7,8)$. Therefore, it became common practice in several sarcoma centers to initiate combination treatment, hypothesizing synergy between gemcitabine and docetaxel. Myelosupression is the primary toxicity (grade 3-4 neutropenia in $17 \%$, grade 3 anemia in $25 \%$ and severe thrombocytopenia in $10 \%$ of the patients) associated with this type of treatment (9). In addition, grade 3 non-hematological toxicities, including fatigue and myalgia, developed in $25 \%$ of the patients. Patients with advanced sarcoma are usually heavily pretreated, with limited bone marrow tolerance and impaired quality of life. Paclitaxel and docetaxel share the same mechanisms of action and were found to exhibit similar efficacies in certain types of cancer, such as breast and lung cancer. Paclitaxel has been proven to be more tolerable when administered weekly, according to studies on breast cancer (10-12). For example, in a prospective randomized trial assessing different schedules and regimens of paclitaxel vs. docetaxel in adjuvant therapy for breast cancer, $71 \%$ of those receiving docetaxel every 3 weeks developed grade 3 or 4 toxicities compared with $28 \%$ of those receiving weekly paclitaxel $(\mathrm{P}=0.001)(11)$. Therefore, we hypothesized that paclitaxel may substitute docetaxel in the combination with gemcitabine for STS and designed a modified weekly protocol of gemcitabine plus paclitaxel $(\mathrm{G} / \mathrm{P})$ to reduce toxicity and maintain the planned schedule. In this study, we report our single institution experience focusing on efficacy and tolerability of modified weekly G/P in 30 patients with advanced STS.

\section{Patients and methods}

Patients and chemotherapy treatment. The medical records of patients with advanced unresectable STS treated between 2002 and 2009 with the G/P protocol were retrospectively evaluated. The G/P protocol included fixed-dose gemcitabine $700 \mathrm{mg} / \mathrm{m}^{2}$ intravenously (i.v.) over $90 \mathrm{~min}$, followed by $70 \mathrm{mg} / \mathrm{m}^{2}$ paclitaxel i.v. over $1 \mathrm{~h}$ on days 1 and 8 of every 3 -week cycle. Our center (Sharett Institute of Oncology, Hadassah-Hebrew University Medical Center, Jerusalem, Israel) served as a 
national referral center. The clinical evaluation upon each admission consisted of an updated medical history focusing on performance status, tolerability and toxicity and a complete blood count. Toxicities were recorded based on the written report of the admitting physician and graded according to the World Health Organization common toxicity criteria (13). The response to treatment was determined according to the Response Evaluation Criteria In Solid Tumors (RECIST) (14) based on computed tomography scan/magnetic resonance imaging scans performed after 2-3 courses of treatment. All the patients provided written informed consent regarding their participation in this study.

\section{Results}

Patient characteristics. A total of 30 patients were treated with G/P for advanced unresectable STS at the Sharett Institute of Oncology between 2002 and 2009. The patient characteristics are summarized in Table I. Half of the patients were treated for high-grade LMS and undifferentiated pleomorphic sarcoma. The vast majority of the patients (27/30) underwent resection of the primary tumor, with one-third (10/27) undergoing R0 resection, 11 received prior adjuvant chemotherapy and 17 patients underwent adjuvant radiotherapy. The majority of the patients (24/30) received a doxorubicin-based regimen prior to G/P. Only 3 patients received G/P as first-line therapy for advanced disease, whereas 21 received G/P as second-line and 6 as third-or further-line therapy. The most common metastatic site was the lung (24 patients) and 14 patients had $\geq 2$ metastatic sites.

Treatment toxicities and response. The 30 patients in our cohort received a total of 190 cycles of G/P. There was no reported treatment-related mortality. The grade 3-4 hematological toxicities included neutropenia in 4 patients (with a single episode of culture-negative neutropenic fever) and anemia in 3 patients $(12 \%)$. Non-hematological toxicity included grade 2 diarrhea (1 patient) and grade 3 sensory neuropathy (1 patient). Postponing treatment and dose reduction were required in 7 patients, which may explain the tolerability.

Clinical outcome. Of the 30 patients, 8 (27\%) achieved a partial response and $10(30 \%)$ experienced stable disease as best response, with an overall clinical benefit of $56 \%$. None of the patients achieved a complete response. The median PFS and OS were 6.1 and 14.3 months, respectively. Of note, all the deceased patients succumbed to progressive disease. As noted above, there was no toxicity-related mortality.

\section{Discussion}

In this retrospective study, the combination of weekly gemcitabine and paclitaxel achieved a partial response rate of $27 \%$ and a disease stabilization rate of $30 \%$ in pretreated patients with advanced STS. The median PFS was 6.2 months and the OS reached 14.3 months. The regimen was well tolerated, without treatment-related mortality. The proportion of grade 3 and 4 hematological toxicities $(\sim 10 \%)$ in patients receiving this protocol was significantly lower compared with patients receiving docetaxel every 3 weeks ( $\sim 6 \%$ incidence of neutropenia) (11).
Table I. Characteristics of the patients $(n=30)$.

\begin{tabular}{|c|c|}
\hline Characteristics & Values \\
\hline Gender (male/female) & $14 / 16$ \\
\hline Median age at diagnosis, years (range) & $56.4(40-70)$ \\
\hline \multicolumn{2}{|l|}{ Initial localization } \\
\hline Extremities & 14 \\
\hline Uterus & 3 \\
\hline Retroperitoneum & 3 \\
\hline Organs other than uterine corpus & 10 \\
\hline \multicolumn{2}{|l|}{ Histology } \\
\hline Leiomyosarcoma & 10 \\
\hline Synovial sarcoma & 1 \\
\hline Liposarcoma & 3 \\
\hline Angiosarcoma & 2 \\
\hline Epithelioid sarcoma & 2 \\
\hline Rhabdomyosarcoma & 1 \\
\hline UPS & 5 \\
\hline Spindle cell sarcoma & 3 \\
\hline Stromal sarcoma & 2 \\
\hline Pleomorphic sarcoma & 1 \\
\hline \multicolumn{2}{|l|}{ Initial FNCLCC grading } \\
\hline I & 1 \\
\hline II & 1 \\
\hline III & 15 \\
\hline Unknown & 13 \\
\hline \multicolumn{2}{|l|}{ Previous treatment } \\
\hline Initial surgery/no surgery & $27 / 3$ \\
\hline R0 & 10 \\
\hline $\mathrm{R} 1$ & 3 \\
\hline $\mathrm{R} 2$ & 3 \\
\hline Unknown & 11 \\
\hline Previous adjuvant chemotherapy & 11 \\
\hline \multicolumn{2}{|l|}{ Lines of chemotherapy prior to $\mathrm{G} / \mathrm{T}$} \\
\hline None & 3 \\
\hline 1 & 21 \\
\hline 2 & 5 \\
\hline$\geq 3$ & 1 \\
\hline Doxorubicin-based chemotherapy & 24 \\
\hline Adjuvant radiation & 17 \\
\hline \multicolumn{2}{|l|}{ Number of metastatic sites } \\
\hline 1 & 16 \\
\hline$\geq 2$ & 14 \\
\hline \multicolumn{2}{|l|}{ Site of metastasis } \\
\hline Lung & 24 \\
\hline Liver & 5 \\
\hline Bone & 6 \\
\hline \multicolumn{2}{|l|}{ Performance status } \\
\hline $0-1$ & 24 \\
\hline 2 & 4 \\
\hline $3-4$ & 2 \\
\hline
\end{tabular}

UPS, undifferentiated pleomorphic sarcoma; FNCLCC, French Federation of Cancer Centers Sarcoma Group; G/T, gemcitabine in combination with paclitaxel. 
To the best of our knowledge, this study is the first to evaluate the efficacy and tolerability of G/P in patients with STS. The combination of taxanes and gemcitabine is increasingly utilized in STS, as well as in bone sarcoma (7-9,15). Early-phase II studies evaluating fixed-dose rate infusion of gemcitabine $900 \mathrm{mg} / \mathrm{m}^{2}$ administered over $90 \mathrm{~min}$ (days 1 and 8) in combination with docetaxel $100 \mathrm{mg} / \mathrm{m}^{2}$ (day 8) every 21 days, achieved objective response rates of $17-36 \%$, with a median time-to-progression of 5.6-6.2 months (4,7). The synergism between the two agentsis of note. The addition of docetaxel to gemcitabine improved outcome compared with single-agent gemcitabine (4). These clinical results were also supported by cell culture data revealing that the sequencing of gemcitabine followed by docetaxel is significant, resulting in superior drug synergism compared with other sequencing (16). Our results with paclitaxel substituting docetaxel suggested equivalent efficacy with significantly reduced toxicity. Although gemcitabine and docetaxel are usually tolerated well when administered as front-line treatment with the support of granulocyte-colony stimulating factor (G-CSF), this combination becomes progressively less tolerable with more advanced lines of treatment. None of the patients in our retrospective cohort received prior G-CSF and, in general, the tendency was to avoid G-CSF and opt for a mild (usually $10 \%$ ) dose reduction instead. With disease progression, pretreatment quality of life and tolerance to treatment become more pertinent to treatment selection.

Only a limited number of chemotherapeutic agents have been found to exhibit some efficacy as single agents in advanced sarcoma. Published studies on single-agent treatments, such as high-dose ifosfamide or temozolamide, reported a limited efficacy $(17,18)$. Trabectedin, which has been approved in Europe for second- or further-line treatment of advanced STS, achieved response rates of $\leq 10 \%$, with significant clinical benefit almost exclusively in LMS and liposarcoma patients (19). Trabectedin lacks cumulative toxicity; however, the first 2-3 cycles may be associated with significant hepatic and hematological toxicity. Pazopanib, a small molecule with vascular endothelial growth factor inhibitory characteristics, may be a viable treatment option for patients with metastatic non-adipocytic STS following chemotherapy. Although tolerance may be improved with dose reductions, pazopanib treatment was shown to stabilize disease in the vast majority of patients, with a limited number of detected responses when measured by RECIST (20).

Patients with STS who have previously received standard chemotherapy, including doxorubicin, ifosfamide, pazopanib, cyclophosphamide and dacarbazine, are faced with a limited number of therapeutic options. Therefore, it is crucial to establish an effective, tolerable protocol for heavily pretreated STS patients, which will control the symptoms and prolong PFS.

There were certain limitations to our study, mainly due to its retrospective design, the limited number of included patients with various histological diagnoses and the potential patient selection bias. In addition, the timing of imaging evaluations was not uniform in our patient population. However, despite these limitations, to the best of our knowledge, this is the first study to describe the efficacy and tolerability of paclitaxel as a substitute to docetaxel in the combination with gemcitabine for the treatment of patients with STS. Our study demonstrated that the combination of gemcitabine and paclitaxel is a tolerable and effective regimen in patients with advanced STSs, particularly after doxorubicin-based regimens. However, these results require confirmation and validation in larger prospective studies.

\section{References}

1. Patel SR, Vadhan-Raj S, Burgess MA, Plager C, Papadopolous N, Jenkins J and Benjamin RS: Results of two consecutive trials of dose-intensive chemotherapy with doxorubicin and ifosfamide in patients with sarcomas. Am J Clin Oncol 21: 317-321, 1998.

2. Lorigan P, Verweij J, Papai Z, et al; European Organisation for Research and Treatment of Cancer Soft Tissue and Bone Sarcoma Group Study: Phase III trial of two investigational schedules of ifosfamide compared with standard-dose doxorubicin in advanced or metastatic soft tissue sarcoma: A European Organisation for Research and Treatment of Cancer Soft Tissue and Bone Sarcoma Group Study. J Clin Oncol 25: 3144-3150, 2007.

3. Pautier P, Floquet A, Penel N, et al: Randomized multicenter and stratified phase II study of gemcitabine alone versus gemcitabine and docetaxel in patients with metastatic or relapsed leiomyosarcomas: a Federation Nationale des Centres de Lutte Contre le Cancer (FNCLCC) French Sarcoma Group Study (TAXOGEM study). Oncologist 17: 1213-1220, 2012.

4. Maki RG, Wathen JK, Patel SR, et al: Randomized phase II study of gemcitabine and docetaxel compared with gemcitabine alone in patients with metastatic soft tissue sarcomas: results of sarcoma alliance for research through collaboration study 002 (corrected). J Clin Oncol 25: 2755-2763, 2007.

5. Merimsky O, Meller I, Flusser G, Kollender Y, Issakov J, Weil-Ben-Arush M, Fenig E, Neuman G, Sapir D, Ariad S, et al: Gemcitabine in soft tissue or bone sarcoma resistant to standard chemotherapy: a phase II study. Cancer Chemother Pharmacol 45: $177-181,2000$

6. Svancárová L, Blay JY, Judson IR, et al: Gemcitabine in advanced adult soft-tissue sarcomas. A phase II study of the EORTC Soft Tissue and Bone Sarcoma Group. Eur J Cancer 38: 556-559, 2002.

7. Hensley ML, Maki R, Venkatraman E, Geller G, Lovegren M, Aghajanian C, Sabbatini P, Tong W, Barakat R and Spriggs DR: Gemcitabine and docetaxel in patients with unresectable leiomyosarcoma: Results of a phase II trial. J Clin Oncol 20: 2824-2831, 2002.

8. Hensley ML: Update on gemcitabine and docetaxel combination therapy for primary and metastatic sarcomas. Curr Opin Oncol 22: 356-361, 2010.

9. Hensley ML, Blessing JA, Mannel R and Rose PG: Fixed-dose rate gemcitabine plus docetaxel as first-line therapy for metastatic uterine leiomyosarcoma: a Gynecologic Oncology Group phase II trial. Gynecol Oncol 109: 329-334, 2008.

10. Wist EA, Sommer HH, Ostenstad B, Risberg T and Fjaestad K: Weekly one-hour paclitaxel as first-line chemotherapy for metastatic breast cancer. Acta Oncol 43: 11-14, 2004.

11. Sparano JA, Wang M, Martino S, Jones V, Perez EA, Saphner T, Wolff AC, Sledge GW Jr, Wood WC and Davidson NE: Weekly paclitaxel in the adjuvant treatment of breast cancer. N Engl J Med 358: 1663-1671, 2008.

12. Kellokumpu-Lehtinen P, Tuunanen T, Asola R, et al: Weekly paclitaxel - an effective treatment for advanced breast cancer. Anticancer Res 33: 2623-2627, 2013.

13. Trotti A, Colevas AD, Setser A, Rusch V, Jaques D, Budach V, Langer C, Murphy B, Cumberlin R, Coleman CN and Rubin P: CTCAE v3.0: development of a comprehensive grading system for the adverse effects of cancer treatment. Semin Radiat Oncol 13: 176-181, 2003.

14. Eisenhauer EA, Therasse P, Bogaerts J, Schwartz LH, Sargent D, Ford R, Dancey J, Arbuck S, Gwyther S, Mooney M, Rubinstein L, Shankar L, Dodd L, Kaplan R, Lacombe D and Verweij J: New response evaluation criteria in solid tumours: revised RECIST guideline (version 1.1). Eur J Cancer 45: 228-247, 2009.

15. Navid F, Willert JR, McCarville MB, Furman W, Watkins A, Roberts W and Daw NC: Combination of gemcitabine and docetaxel in the treatment of children and young adults with refractory bone sarcoma. Cancer 113: 419-425, 2008.

16. Leu KM, Ostruszka LJ, Shewach D, Zalupski M, Sondak V, Biermann JS, Lee JS, Couwlier C, Palazzolo K and Baker LH: Laboratory and clinical evidence of synergistic cytotoxicity of sequential treatment with gemcitabine followed by docetaxel in the treatment of sarcoma. J Clin Oncol 22: 1706-1712, 2004. 
17. Sutton GP, Blessing JA, DeMars LR, Moore D, Burke TW and Grendys EC: A phase II Gynecologic Oncology Group trial of ifosfamide and mesna in advanced or recurrent adenocarcinoma of the endometrium. Gynecol Oncol 63: 25-27, 1996.

18. Talbot SM, Keohan ML, Hesdorffer M, Orrico R, Bagiella E, Troxel AB and Taub RN: A phase II trial of temozolomide in patients with unresectable or metastatic soft tissue sarcoma. Cancer 98: 1942-1946, 2003.

19. Sanfilippo R, Grosso F, Jones RL, Banerjee S, Pilotti S, D'Incalci M, Dei Tos AP, Raspagliesi F, Judson I and Casali PG: Trabectedin in advanced uterine leiomyosarcomas: a retrospective case series analysis from two reference centers. Gynecol Oncol 123: 553-556, 2011.
20. van der Graaf WT, Blay JY, Chawla SP, et al; EORTC Soft Tissue and Bone Sarcoma Group; PALETTE study group: Pazopanib for metastatic soft-tissue sarcoma (PALETTE): a randomised, double-blind, placebo-controlled phase 3 trial. Lancet 379: 1879-1886, 2012. 